13,03

\title{
Электронная структура термически окисленного вольфрама
}

\author{
() П.А. Дементьев ${ }^{1}$, Е.В. Дементьева ${ }^{1}$, М.Н. Лапушкин ${ }^{1,}$, Д.А. Смирнов ${ }^{2}$, С.Н. Тимошнев ${ }^{3}$ \\ ${ }^{1}$ Физико-технический институт им. А.Ф. Иофффе РАН, \\ Санкт-Петербург, Россия \\ ${ }^{2}$ Institut für Festkörper- und Materialphysik, Technische Universität Dresden, \\ 01062 Dresden, Germany \\ ${ }^{3}$ Санкт-Петербургский национальный исследовательский Академический университет им. Ж.И. Алфёрова РАН, \\ Санкт-Петербург, Россия \\ ๑E-mail: lapushkin@ms.ioffe.ru
}

Поступила в Редакцию 25 марта 2021 г.

В окончательной редакции 30 марта 2021 г.

Принята к публикации 30 марта 2021 г.

С помощью метода фотоэлектронной спектроскопии проведены исследования in situ в сверхвысоком вакууме электронной структуры чистой поверхности вольфрама, окисленного при давлении кислорода 1 Torr и температуре $1000 \mathrm{~K}$. Изучены спектры фотоэмиссии из валентной зоны и остовных уровней $\mathrm{O} 1 s$, $\mathrm{O} 2 s$, W $4 f$ при синхротронном возбуждении в диапазоне энергий фотонов $80-600 \mathrm{eV}$. Найдено, что формируется полупроводниковая пленка окисла вольфрама, которая содержит различные окислы вольфрама со степенью окисления от $6+$ до 4+. На поверхности образуются в основном окислы вольфрама со степенью окисления 6+, доля которых постепенно уменьшается по мере удаления от поверхности с увеличением окислов вольфрама со степенью окисления $4+$.

Ключевые слова: окисление вольфрама, фотоэлектронная спектроскопия.

DOI: $10.21883 /$ FTT.2021.08.51173.065

\section{1. Введение}

Окисел вольфрама $\mathrm{WO}_{3}$ нашел широкое применение в электронике, газовых сенсорах, электро- и фотохромных системах, солнечных элементах, батареях, катализаторах окислительных (восстановительных) реакций, эмиттерах при термической ионизации атомов и органических молекул и т.д. Существуют разные методы получения пленок окислов вольфрама: магнетронное испарение, термическое испарение, золь-гель-осаждение, газофазное осаждение, жидкофазные способы, включая электрохимический синтез, и т.д. [1-3]. Также можно получить тонкие пленки окисла вольфрама при его прогреве в кислороде.

Известно, что оксид молибдена $\mathrm{WO}_{3}$ имеет следующие основные фазы: моноклинную II $\varepsilon$-фазу $(T<230 \mathrm{~K})$, моноклинную І стабильную $\gamma$-фазу $(230<T<603 \mathrm{~K})$, триклинную $\delta$-фазу $(230<T<290 \mathrm{~K})$, стабильную орторомбическую фазу $\beta$-фазу $(603<T<1013 \mathrm{~K})$, и тетрагональную $\alpha$-фазу $(T>1013 \mathrm{~K})$ [4-5]. Также существует ряд субстехиометрических оксидов вольфрама $\mathrm{W}_{n} \mathrm{O}_{3 n-1}$ и $\mathrm{W}_{n} \mathrm{O}_{3 n-2}$, которые образуются при перестройке кристаллической структуры за счет меньшего количества кислорода [6]. Кристаллическая структура $\mathrm{WO}_{3}$ образована 1 атомом вольфрама и 6 атомами кислорода, которые образуют октаэдр, который незначительно деформируется для разных фаз $\mathrm{WO}_{3}$. Для $\gamma$-моноклинной фазы $\mathrm{WO}_{3}$ термодинамически стабильной является реконструированная поверхность (001) [7].
$\mathrm{WO}_{3}$ - это широкозонный полупроводник $n$-типа с шириной запрещенной зоны $\sim 2.8 \mathrm{eV}$. Существует ряд расчетов электронной структуры $\mathrm{WO}_{3}$ методом функционала плотности, например [8-12], в которых было показано, что для моноклинной фазы $\mathrm{WO}_{3}$ валентная зона сформирована из состояний О $2 p$ с незначительной примесью W $5 d$-состояний, а зона проводимости образована W $5 d$-состояниями. В [8] получено, что валентная зона представляет собой широкую зону $\sim 8 \mathrm{eV}$ с двумя пиками с энергией связи $E_{b}=1$ и $4 \mathrm{eV}$ ниже положения максимума валентной зоны $E_{\mathrm{VBM}}$. Похожие результаты были получены и в других работах [9-12].

Электронная структура окислов вольфрама хорошо изучена для образцов, полученных различными способами. Работы, в основном, направлены на изучение, как формы фотоэлектронных спектров остовных уровней вольфрама $\mathrm{W} 4 f$, так и спектра валентной зоны, которые зависят как от внешних условий, так и методов приготовления [13-17]. Форма спектров остовных уровней вольфрама $\mathrm{W} 4 f$ хорошо отражает состояния вольфрама с разными степенями окисления. Существенно меньше внимания уделено изучению остовных состояний $\mathrm{O} 1 s$ $(\mathrm{O} 2 s)$, из которых можно выделить влияние адсорбированной воды на формирование окислов вольфрама [15].

В [13] исследовано формирование пленок окисла вольфрама при магнетронном распылении вольфрама в атмосфере $\mathrm{Ar}-\mathrm{O}_{2}$ и показано, что уменьшение давления кислорода от $2.4 \cdot 10^{-2} \mathrm{mbar}$ до $2.4 \cdot 10^{-3} \mathrm{mbar}$ приводит к увеличению ширины спектра остовных состояний $\mathrm{W} 4 f$ и появлению новых пиков. Так, для давления кислорода 
$2.4 \cdot 10^{-2}$ mbar в спектре присутствует только состояния вольфрама с высшей степенью окисления $\mathrm{W}^{6+}$. А при уменьшении давления кислорода до $2.4 \cdot 10^{-3} \mathrm{mbar}$ в спектре можно выделить уже три состояния вольфрама с разными степенями окисления: $\mathrm{W}^{6+}, \mathrm{W}^{5+}$ и $\mathrm{W}^{4+}$. Изменение давления кислорода не оказывает влияния на форму спектра О $1 s$. Форма спектра валентной зоны остается без изменений, за исключением возрастания доли W $5 d$-состояний вблизи уровня Ферми $\left(E_{\mathrm{F}}\right)$. В $[14]$ исследованы пленки $\mathrm{WO}_{3}$, полученные при термическом напылении порошка $\mathrm{WO}_{3}$. Спектр остовных состояний $\mathrm{W} 4 f$ соответствует состоянию вольфрама $\mathrm{W}^{6+}$. Спектр валентной зоны широкий $\sim 12 \mathrm{eV}$ с максимумом при $6.3 \mathrm{eV}$. При энергии возбуждения соответствующей минимальной глубине выхода фотоэлетронов $(h v=50 \mathrm{eV})$ вблизи уровня Ферми наблюдаются небольшие пики W $5 d$. В [15] моноклинные пленки $\mathrm{WO}_{3}$ были получены при пиролизном распылении вольфрамата аммония. В спектре остовных состояний $\mathrm{W} 4 f$ наблюдаются два состояния вольфрама $-\mathrm{W}^{6+}$ и $\mathrm{W}^{5+}$. В спектре остовных состояний $\mathrm{O} 1 s$ выделено два пика: один соответствует $\mathrm{O}^{2-}(530.69 \mathrm{eV})$, а второй соответствует кислороду в составе гидроксила $(532.62 \mathrm{eV})$. В [16] исследовано формирование моноклинных пленок окисла вольфрама при магнетронном распылении вольфрама в атмосфере $\mathrm{Ar-} \mathrm{O}_{2}$. Спектр остовных состояний $\mathrm{W} 4 f$ для свеженапыленных пленок соответствуют состоянию вольфрама $\mathrm{W}^{6+}$. Прогрев пленок приводит к сдвигу положения максимумов пиков $\mathrm{W} 4 f$ и, по мнению авторов, это связано с формированием соединений с дефицитом кислорода $\mathrm{W}_{18} \mathrm{O}_{49}, \mathrm{~W}_{24} \mathrm{O}_{68}$ и(или) $\mathrm{WO}_{2}$.

Как было указано выше, при исследованиях электронной структуры окисных пленок $\mathrm{WO}_{3}$ в созданных пленках иногда наблюдаются состояния вольфрама со степенью окисления 4+. Однако исследований непосредственно $\mathrm{WO}_{2}$ существенно меньше, чем $\mathrm{WO}_{3}$, что определяется его меньшим применением в технологиях. $\mathrm{B}$ отличии от $\mathrm{WO}_{3}$ окисел $\mathrm{WO}_{2}$ является металлом. Известен ряд фаз $\mathrm{WO}_{2}$ : стабильная моноклинная фаза и метастабильная орторомбическая для больших температур [18-20]. В [21] расчет электронной плотности состояний для обоих фаз показывает, что валентная зона вблизи уровня Ферми сформирована О $2 p$ - и $\mathrm{W} 5 d$-состояниями, зона с энергией связи больше $2.5 \mathrm{eV}$ сформирована в основном О $2 p$-состояниями с небольшой примесью W $5 d$-состояний. При этом спектр валентной зоны широкий $-10 \mathrm{eV}$. В [22] был проведен расчет методом функционала плотности электронных состояний моноклинной структуры металлического $\mathrm{WO}_{2}$ и показано, что валентная зона вблизи уровня Ферми сформирована W $5 d$-состояниями с двумя пиками, а зона с энергией связи больше $2.5 \mathrm{eV}$ сформирована в основном О 2p-состояниями. В [23] были исследованы пленки $\mathrm{WO}_{2}$, полученные при окислении вольфрама в $\mathrm{Ar}_{2} \mathrm{O}_{2}$ смеси при магнетронном распылении на подложку $\mathrm{Al}_{2} \mathrm{O}_{3}$. В спектре широкой валентной зоны около уровня Ферми наблюдается два узких W $5 d$-пика в области
$0.8-2.0 \mathrm{eV}$, что совпадает с расчетом [22], и максимум при $\sim 7 \mathrm{eV}$. Однако в спектре остовных состояний $\mathrm{W} 4 f$ четко видны состояния $\mathrm{W}^{6+}$ и $\mathrm{W}^{4+}$. Похожие результаты были получены и в более ранних работах [24-25].

Процесс окисления вольфрама был исследован в многочисленных работах, например, [26-28]. Однако до сих пор нет однозначной картины, какие окислы вольфрама и при каких температурах образуются. Толщина окисной пленки зависит от температуры, давления кислорода и влажности. При температурах до 600-673 К образуется окисел $\mathrm{WO}_{3}$, который образует защитную голубую пленку. При повышении температуры свыше $780 \mathrm{~K}$ пленка окисла растрескивается и за счет диффузии кислорода происходит образование смешанных окислов $\mathrm{WO}_{2.75}$ или $\mathrm{WO}_{2.92}$. При температурах более $880 \mathrm{~K}$ формируется окисная пленка $\mathrm{WO}_{3}$. При $T>1000 \mathrm{~K}$ наблюдается сублимация окислов вольфрама и при $>1200 \mathrm{~K}$ происходит быстрое разрушение пленки окисла. При $T=1550 \mathrm{~K}$ на поверхности вольфрама может образовываться только монослойная пленка кислорода [29].

Целью настоящей работы было исследование электронной структуры поверхности окисленного W, полученного при термическом окислении W при давлении кислорода 1 Torr и $T=950 \mathrm{~K}$. Этим условиям соответствует методика создания эмиттеров из окисленного вольфрама, используемых в термической ионизации атомов и молекул [30].

\section{2. Эксперимент}

В настоящей работе процесс окисления предварительно отожженного в вакууме вольфрама был проведен при температуре $950 \mathrm{~K}$ при давлении кислорода 1 Torr в течение $2 \mathrm{~h}$. Фотоэмиссионные исследования были выполнены в Российско-Германской лаборатории на синхротроне HZB BESSY II (Берлин, Германия) с использованием метода фотоэлектронной спектроскопии (ФЭС) при возбуждении в диапазоне энергий фотонов 80-600 eV. Регистрировались фотоэлектроны по нормали к поверхности, возбуждающий пучок падал на поверхность образца под углом $45^{\circ}$. Исследования окисленной тонкой пленки вольфрама проводились in situ в вакууме $P<5 \cdot 10^{-10}$ Torr при комнатной температуре. Регистрировались спектры фотоэмиссии в области валентной зоны, а также спектры остовных уровней $\mathrm{O} 1 s$, $\mathrm{O} 2 s, \mathrm{~W} 4 f$. Полное энергетическое разрешение было $50 \mathrm{meV}$. Для всех приведенных ниже спектров фон был вычтен по методу Ширли. Перед фотоэмиссионными исследованиями образцы подвергались отжигу в высоком вакууме при температуре $\sim 900 \mathrm{~K}$.

\section{3. Экспериментальные результаты и обсуждение}

На рис. 1 приведены спектры фотоэмиссии остовного уровня дублета $\mathrm{W} 4 f_{5 / 2}$ и $\mathrm{W} 4 f_{7 / 2}$ для чистой поверх- 
Энергия связи уровней $\mathrm{W} 4 f_{5 / 2}\left(E_{b}\right)$, ширина пика на полувысоте $(\Gamma)$ и относительная площадь $(A)$, занимаемая пиком для поверхности окисленного вольфрама

\begin{tabular}{c|c|c|c|c|c|c|c|c|c|c}
\hline Параметр & $\mathrm{W}^{4+}$ & $\mathrm{W}^{5+}$ & $\mathrm{W}^{6+}$ & $\mathrm{W}^{4+}$ & $\mathrm{W}^{5+}$ & $\mathrm{W}^{6+}$ & $\mathrm{W}^{4+}$ & $\mathrm{W}^{5+}$ & $\mathrm{W}^{6+}$ \\
\hline$h v, \mathrm{eV}$ & \multicolumn{3}{|c|}{100} & \multicolumn{3}{|c}{600} \\
\hline$E_{b}, \mathrm{eV}$ & 33.05 & 34.31 & 35.74 & 33.05 & 34.30 & 35.78 & 33.04 & 34.36 & 36.08 \\
\hline$\Gamma, \mathrm{eV}$ & 0.68 & 1.12 & 2.22 & 0.70 & 1.14 & 2.2 & 1.32 & 1.56 & 2.32 \\
\hline$A, \%$ & 11 & 22 & 67 & 11 & 22 & 67 & 25 & 27 & 48
\end{tabular}

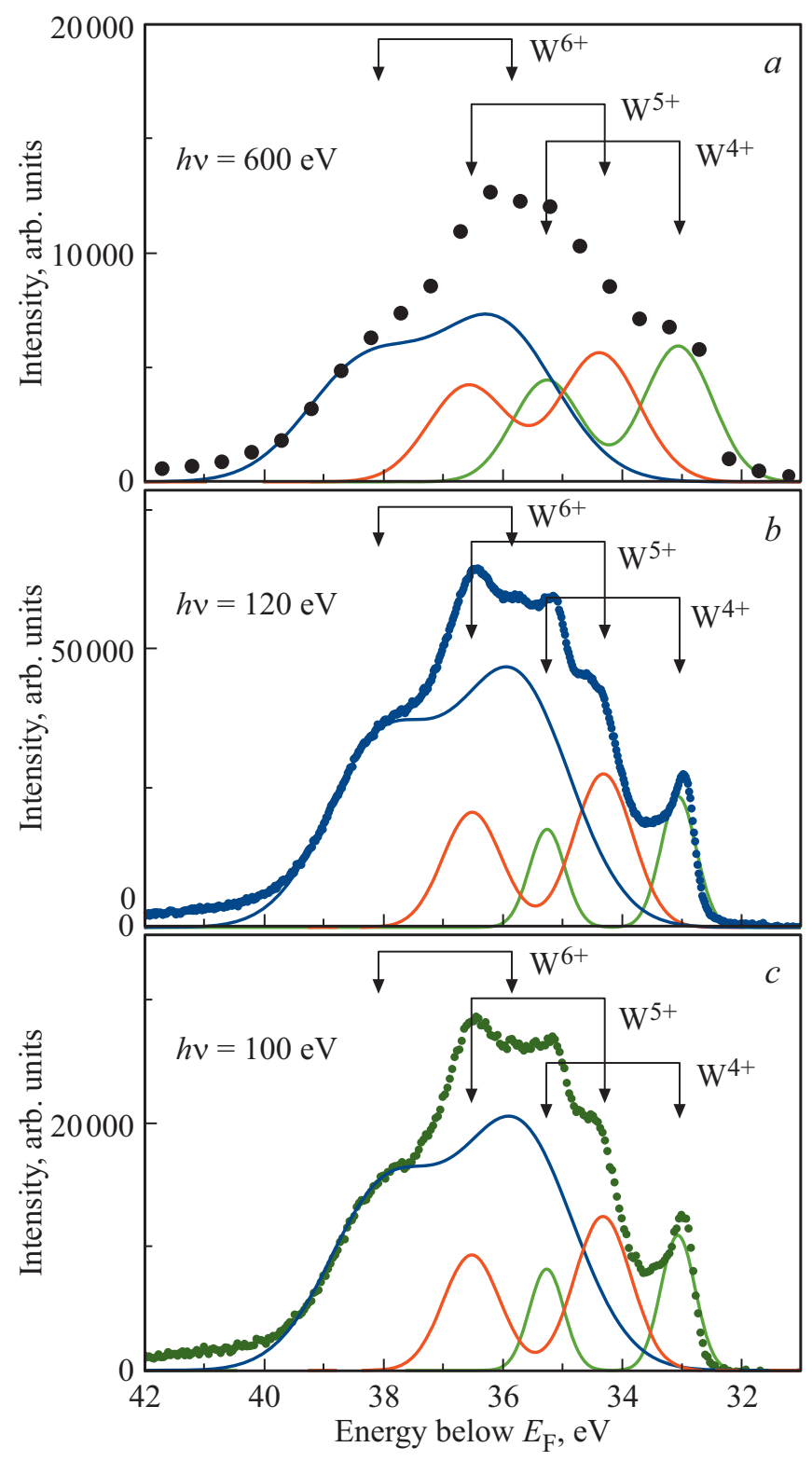

Рис. 1. Анализ нормированного спектра фотоэмиссии остовного уровня $\mathrm{W} 4 f$ для поверхности окисленного вольфрама при энергии возбуждения $h v=600 \mathrm{eV}(a), h v=120 \mathrm{eV}(b)$ и $h v=100 \mathrm{eV}(c)$. Точками приведен экспериментальный спектр, толстыми сплошными линиями приведен вклад $\mathrm{W}^{6+}, \mathrm{W}^{5+}$ и $\mathrm{W}^{4+}$ состояний. ности окисленного вольфрама для трех энергий возбуждения: $h v=100 \mathrm{eV}, h v=120 \mathrm{eV}$ и $h v=600 \mathrm{eV}$ (показаны точками). В спектрах четко видно до пяти пиков. Наблюдаемые пики можно связать уровнями дублета $\mathrm{W} 4 f_{5 / 2}$ и $\mathrm{W} 4 f_{7 / 2}$, которые можно приписать различным степеням окисления вольфрама от 4 до 6, что совпадает с результатами [13]. Пиков, относящихся к $\mathrm{W}^{0}$, которые должны быть расположены при $E_{b}=31.4 \mathrm{eV}$ и $33.6 \mathrm{eV}$, в спектре не обнаружено. Видно, что с увеличением энергии возбуждения форма спектра изменяется, это связано с тем, что чем больше кинетическая энергия электронов, тем больше глубина выхода фотоэлектронов. Т.е. при $h v=100 \mathrm{eV}$ фотоэлектроны выходят из приповерхностной области, а при $h v=600 \mathrm{eV}$ фотоэлектроны вылетают из глубины пленки. Расстояние между уровнями в дублете составляет $2.2 \mathrm{eV}$. Было проведено разложение экспериментального спектра парами функций Гаусса с спектральным расщеплением равным $2.2 \mathrm{eV}$ и соотношением интенсивностей 4:3. Результаты разложения также приведены на рис. 1. Полученные параметры разложения экспериментального пика $\mathrm{W} 4 f$ на составляющие: положение максимумов интенсивности с энергией связи $\left(E_{b}\right)$ относительно уровня Ферми, полная ширина на полувысоте пика $(\Gamma)$ и относительная площадь $(A)$ под пиком в процентах приведены в таблице.

Из таблицы видно, что положение пиков практически не изменяется, а происходит только изменение ширины пика Г и относительной площади для каждого компонента $A$. При энергии возбуждения $h v=100$ и $120 \mathrm{eV}$ соотношение компонент $A_{\mathrm{W} 6+}: A_{\mathrm{W} 5+}: A_{\mathrm{W} 4+}$ равно $67: 22: 11$, а для энергии возбуждения $h v=600 \mathrm{eV}$ соотношение этих компонент равно 48:27:25. Так как с увеличением энергии возбуждения фотоэмиссии глубина выхода фотоэлектронов возрастает, то такое изменение соотношения указывает на то, что на поверхности больше концентрация вольфрама с большей степенью окисления. Такая разница вызвана диффузионными процессами кислорода вглубь образца при окислении вольфрама. Также видно, что с увеличением глубины выхода фотоэлектронов увеличивается и ширина пиков. Это может указывать на присутствие окислов и других степеней окисления, что совпадает с известным фактом большого количества различных стехиометрий окислов и фаз окислов воль- 

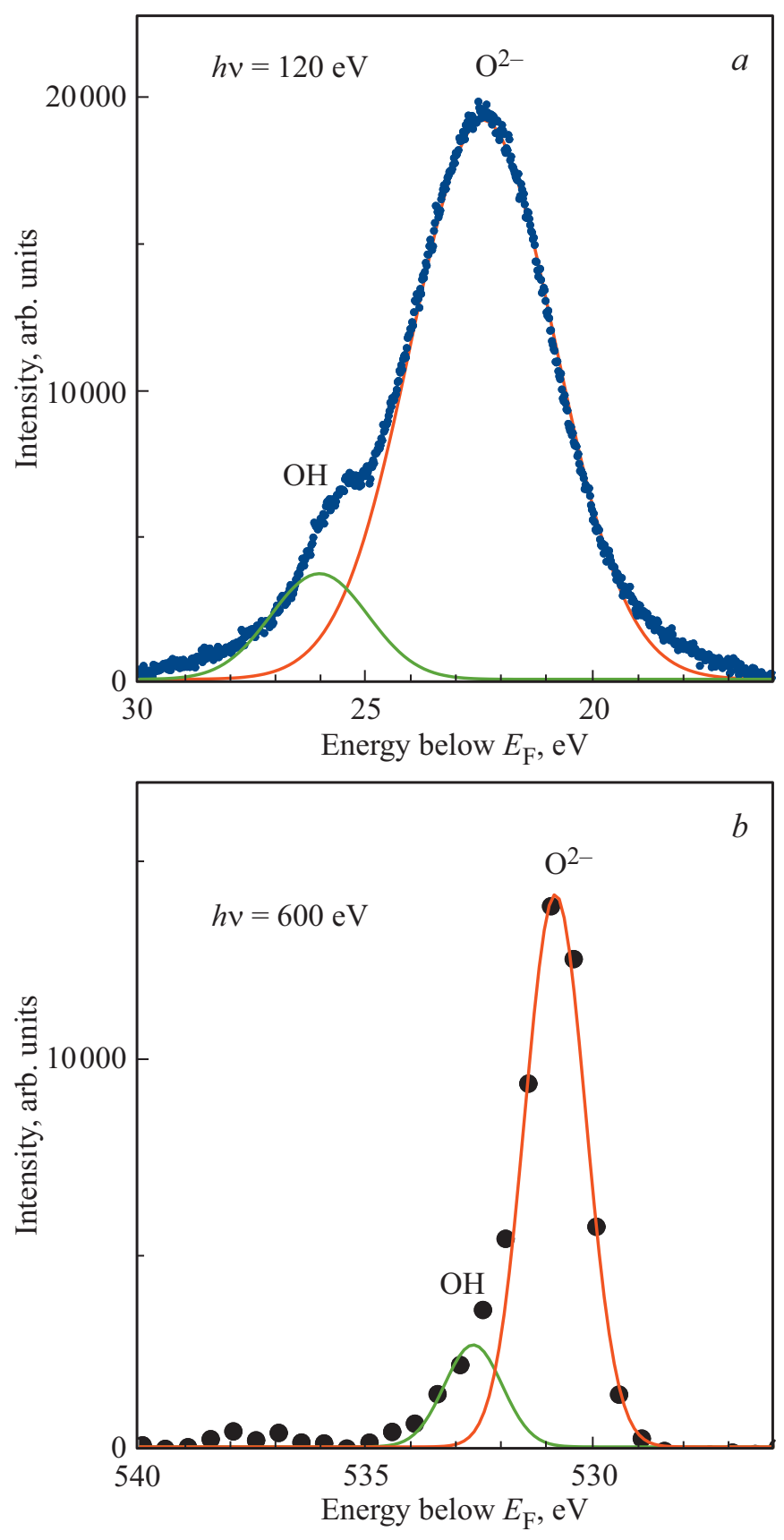

Рис. 2. Спектры нормальной фотоэмиссии остовных уровней $\mathrm{O} 2 s(a)$ и О $1 s(b)$ для поверхности окисленного вольфрама. Энергия возбуждения $h v=120 \mathrm{eV}(a)$ и $h v=600 \mathrm{eV}(b)$.

фрама [4-7,13-16]. Большая ширина пика $\mathrm{W}^{6+}$ может быть связана с наличием в окисле не только $\mathrm{WO}_{3}$, но и других субстехиометрических окислов.

На рис. 2 представлены спектры фотоэмиссии из остовных уровней $\mathrm{O} 2 s \quad(h v=120 \mathrm{eV})$ и $\mathrm{O} 1 s$ $(h v=600 \mathrm{eV})$ для поверхности окисленного вольфрама. Спектр фотоэмиссии при энергии возбуждения $h v=120 \mathrm{eV}$ из остовного уровня $\mathrm{O} 2 s$ соответствует поверхностной фотоэмиссии, так как кинетическая энергия фотоэлектронов близка к минимуму выхода на универ- сальной кривой выхода фотоэлектронов от кинетической энергии [31]. Спектр фотоэмиссии при энергии возбуждения $h v=600 \mathrm{eV}$ остовного уровня $\mathrm{O} 1 s$ отражает выход из глубины пленки, т.н. объемной фотоэмиссии. При разложении спектра можно выделить два пика при $E_{B}=530.8$ и $532.8 \mathrm{eV}$ с шириной пиков $1.50 \mathrm{eV}$. Эти пики можно связать с двумя состояниями кислорода: в составе окисла и гидроксила, как это было получено в [15]. Соотношение этих компонент 7:1. Похожий результат был получен при энергии возбуждения $h v=120 \mathrm{eV}$ в спектре остовного состояния $\mathrm{O} 2 s$ (фотоэмиссия из поверхности окисла), в котором четко наблюдаются два пика с энергией связи при $E_{B}=22.4$ и $26.0 \mathrm{eV}$ с возросшей шириной пиков до 3.6 и $2.4 \mathrm{eV}$, соответственно. Возрастание ширины пиков можно связать с большим вкладом на поверхности окислов вольфрама со степенью окисления близкой к $6+$. Соотношение этих компонент 9:1. Наличие гидроксила на поверхности и в глубине окисла вполне объяснимо присутствием паров воды в процессе окисления вольфрама. Эти результаты совпадают с данными, полученными при

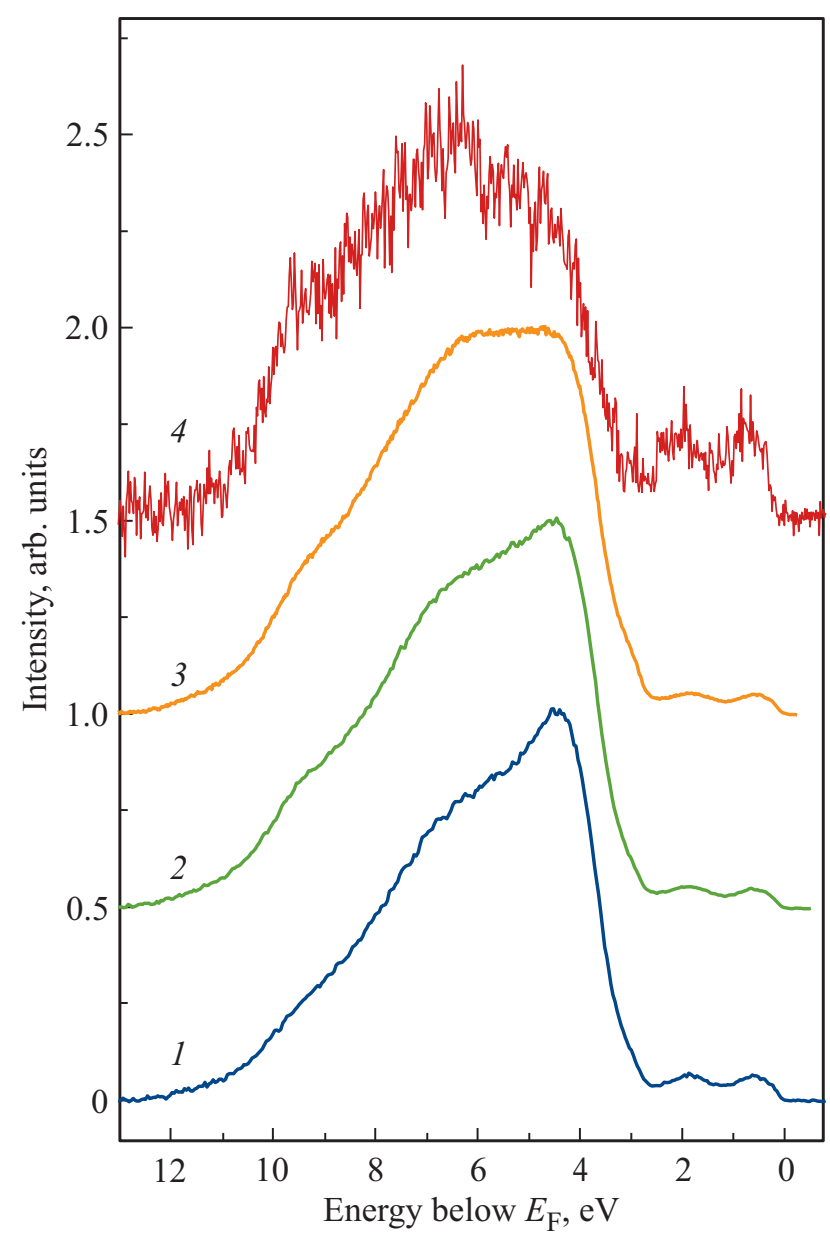

Рис. 3. Нормированные спектры фотоэмиссии в области валентной зоны для поверхности окисленного вольфрама при различных энергиях возбуждения: $1-h v=80 \mathrm{eV}, 2-$ $h v=100 \mathrm{eV}, 3-h v=120 \mathrm{eV}, 4-h v=600 \mathrm{eV}$. 
исследовании формирования термически окисленного молибдена [32].

Нормированные спектры фотоэмиссии в области валентной зоны окисленного вольфрама представлены на рис. 3 для энергий возбуждения в диапазоне от 80 до $600 \mathrm{eV}$. Спектры приведены к положению уровня Ферми на поверхности $\left(E_{\mathrm{F}}\right)$, который определяется линейной аппроксимацией низкоэнергетического края спектра валентной зоны подложки из нержавеющей стали, с которой образцы были в омическом контакте. В области валентной зоны спектр окисленного вольфрама не имеет особенностей и имеет ширину $\sim 12 \mathrm{eV}$. Фотоэлектронные спектры валентной зоны термически окисленного $\mathrm{W}$ на воздухе совпадают с хорошо известными спектрами валентной зоны окисленного вольфрама $[13,14]$. Пик валентной зоны не имеет особенностей и его максимум расположен при $4.5-6.5 \mathrm{eV}$ ниже $E_{\mathrm{F}}$ и чем больше энергия возбуждения, тем дальше от уровня Ферми он расположен. Также имеется небольшое плечо в области энергии связи больших, чем положение максимума пика валентной зоны. Слабо выраженный максимум при $E_{b}=6.6 \mathrm{eV}$ для $h v=80 \mathrm{eV}$ совпадает с положением максимума пика валентной зоны для $h v=600 \mathrm{eV}$, это связано с тем, что в глубине окисленного вольфрама возрастает доля окисла с меньшей степенью окисления $4+$, как это видно из спектров уровней $\mathrm{W} 4 f$. Можно предположить, что спектр валентной зоны определяется вкладом окислов $\mathrm{WO}_{3}$.

Интенсивность пиков с $E_{b}=0.6$ и $2.0 \mathrm{eV}$, которые можно приписать $\mathrm{W} 5 d$-состояниям, возрастает с увеличением энергии возбуждения, что отражает возрастание доли окисла $\mathrm{WO}_{2}$ с увеличением глубины зондирования. Это совпадает с результатами работ [33,34], где было показано уменьшение пиков состояний $\mathrm{W} 5 d$ вблизи уровня Ферми по мере формирования окисла $\mathrm{WO}_{3}$.

\section{4. Выводы}

Исследована электронная структура термически окисленного вольфрама. Показано, что на поверхности вольфрамовой подложки формируются окислы вольфрама с преобладающим вкладом окисла вольфрама со степенью окисления $6+$. По мере удаления от поверхности увеличивается доля окисла вольфрама со степенью окисления $4+$, что может быть связано как с недостаточным давлением кислорода при окислении, так и временем окисления. Наличие незначительного количества кислорода в составе гидроксила связывается с присутствием паров воды при окислении. Спектр валентной зоны соответствует полупроводниковому характеру окисла вольфрама.

\section{Финансирование работы}

Исследование выполнено при финансовой поддержке РФФИ в рамках научного проекта № 20-02-00370.
Исследовательский проект был поддержан РоссийскоГерманской лабораторией на BESSY II. Мы благодарим HZB за выделение времени пучка синхротронного излучения.

\section{Конфликт интересов}

Авторы заявляют, что у них нет конфликта интересов.

\section{Список литературы}

[1] H. Zheng, J.Z. Ou, M.S. Strano, R.B. Kaner, A. Mitchell, K. Kalantar-zadeh. Adv. Funct. Mater. 21, 2175 (2011).

[2] V.R. Buch, A.K. Chawla, S.K. Rawal. Appl. Sci. Lett. 1, 115 (2015).

[3] C.C. Mardare, A.W. Hassel. Phys. Status Solidi A 216, 1900047 (2019).

[4] E.K.H. Salje, S. Rehmann, F. Pobell, D. Morris, K.S. Knight, T. Herrmannsdörfer, M.T. Dove. J. Phys. Condens. Matter 9, 6563 (1997).

[5] T. Vogt, P.M. Woodward, B.A. Hunter. J. Solid State Chem. 144, 209 (1999).

[6] H.A. Wriedt. Bull. Alloy Phase Diag. 10, 368 (1989).

[7] P.M. Oliver, S.C. Parker, R.G. Egdell, F.H. Jones. J. Chem. Soc., Faraday Trans. 92, 2049 (1996).

[8] F. Wang, C. Di Valentin, G. Pacchioni. J. Phys. Chem. C 115, 8345 (2011).

[9] J. Tanga, J. Ye. J. Mater. Chem. 15, 4246 (2005).

[10] M.B. Johansson, G. Baldissera, I. Valyukh, C. Persson, H. Arwin, G.A. Niklasson, L. Österlund. J. Phys: Condens. Matter 25, 205502 (2013).

[11] H. Hamdi, E.K.H. Salje, P. Ghosez, E. Bousquet. Phys. Rev. B 94, 24 (2016).

[12] I. Perez, J.C.M. Faudoa, J.R.A. Acuna, J.T.E. Galindo. Comput. Mater. Sci. 190, 110248 (2021).

[13] O. Bouvard, A. Krammer, A. Schüler. Surf. Interface Anal. 48, 660 (2016).

[14] L. Ottaviano, F. Bussolotti, L. Lozzi, M. Passacantando, S. La Rosa, S. Santucci. Thin Solid Films 436, 9 (2003).

[15] V.V. Ganbavle, S.V. Mohite, J.H. Kim b, K.Y. Rajpure. Current Appl. Phys. 15, 84 (2015).

[16] H. Simchi, B.E. McCandless, T. Meng, W.N. Shafarman. J. Alloys Comp. 617, 609 (2014).

[17] Y.K. Park, C.J. Lim, Y. JiIm, S. Cho, S.W. Cho, H. Lee, H. Ogasawara. Current Appl. Phys. 21, 31 (2021).

[18] D.J. Palmer, P.G. Dickens. Acta Crystallogr. B 35, 2199 (1979).

[19] A.A. Bolzan, B.J. Kennedy, C.J. Howard. Aust. J. Chem. 48, 1473 (1995).

[20] M.R. Sundberg, P.-E. Werner, I.P. Zibrov. Z. Kristallogr. 209, 662 (1994).

[21] V.L. Shaposhnikov, D.B. Migas, V.N. Rodin, V.E. Borisenko. Phys. Status Solidi B 248, 1471 (2011).

[22] N. Smolentsev, M. Sikora, A.V. Soldatov, K.O. Kvashnina, P. Glatzel. Phys. Rev. B 84, 235113 (2011).

[23] K. Fujiwara, A. Tsukazaki. J. Appl. Phys. 125, 085301 (2019).

[24] A. Gulino, S. Parker, F.H. Jones, R.G. Egdell. J. Chem. Soc., Faraday Trans. 92, 2137 (1996).

[25] F.H. Jones, R.G. Egdell, A. Brown, F.R. Wondre. Surf. Sci. 374, 80 (1997). 
[26] C S.C. Cifuentes, M.A. Monge, P. Pe. Corrosion Sci. 57, 114 (2012).

[27] E.A. Gulbransen, K.F. Andrew. J. Electrochem. Soc. 107, 619 (1960).

[28] C.J. Rosa, G.C. Chen, V.K. Sikka. Z. Metallk. 71, 529 (1980).

[29] В.Н. Агеев, Н.И. Ионов. ФТТ 11, 3200 (1969).

[30] U.Kh. Rasulev, E.Ya. Zandberg. Prog. Surf. Sci. 28, 181 (1988).

[31] I. Lindau, W.E. Spicer. J. Electr. Spectroscopy 3, 409 (1974).

[32] R. Sohal, C. Walczyk, P. Zaumseil, D. Wolansky, A. Fox, B. Tillack, H.-J. Müssig, T. Schroeder. Thin Solid Films 517, 4534 (2009).

[33] F.J. Wonga, S. Ramanathan. Mater. Res. 28, 2555 (2013).

[34] K. Fujiwara, A. Tsukazaki, J. Appl. Phys. 125, 085301 (2019).

Редактор К.В. Емиев 